\title{
Prevalência de Burnout em profissionais de saúde no enfrentamento da Covid-19: uma revisão sistemática
}

\author{
Burnout prevalence in health professionals facing Covid-19: a systematic review \\ Prevalencia del Burnout en los profesionales sanitarios que se enfrentan a Covid-19: una revisión \\ sistemática
}

Recebido: 19/11/2021 | Revisado: 27/11/2021 | Aceito: 28/11/2021 | Publicado: 10/12/2021

\author{
Jadon Araújo Macêdo Silva \\ ORCID: https://orcid.org/0000-0002-4759-549X \\ Universidade Federal da Paraíba, Brasil \\ E-mail: jadonmacedo@gmail.com \\ Luís Eduardo Oliveira da Silva \\ ORCID: https://orcid.org/0000-0003-0333-2361 \\ Universidade Federal da Paraíba, Brasil \\ E-mail: oliveiraluisseduardo@gmail.com \\ Ywkiane Lopes de Araújo \\ ORCID: https://orcid.org/0000-0003-0326-9841 \\ Universidade Federal da Paraíba, Brasil \\ E-mail: ywkiane1@gmail.com \\ Wallace Entringer Bottacin \\ ORCID: https://orcid.org/0000-0001-7721-5876 \\ Universidade Federal do Paraná, Brasil \\ E-mail: wallace.bottacin@gmail.com \\ Thais Teles de Souza \\ ORCID: https://orcid.org/0000-0002-4672-9249 \\ Universidade Federal da Paraíba, Brasil \\ E-mail: thaisteles3@gmail.com \\ Walleri Christini Torelli Reis \\ ORCID: https://orcid.org/0000-0001-6911-4792 \\ Universidade Federal da Paraíba, Brasil \\ E-mail: walleri.ctr@gmail.com
}

\begin{abstract}
Resumo
O presente trabalho realizou um levantamento da prevalência da Síndrome de Burnout (SB) em profissionais de saúde, que estiveram na linha de frente do enfrentamento da pandemia de COVID-19. O processo metodológico seguiu as recomendações PRISMA e COCHRANE e as etapas de triagem, elegibilidade e extração de dados foram realizadas por revisores independentes, em planilhas padronizadas. Após esse processo, 26 estudos transversais foram incluídos. Os principais desfechos encontrados corroboram para a variação dos escores médios nas três dimensões do burnout de acordo com a área profissional, local e público acometido. A maioria dos estudos apontaram os profissionais da enfermagem como as populações mais afetadas e com maior prevalência de escores compatíveis nas três dimensões da SB, sendo o público do sexo feminino até $60 \%$ mais acometido que o masculino. Esses dados permitem inferir que medidas mais eficazes precisam ser tomadas em relação ao bem-estar mental desses profissionais. Medidas relacionadas com a infraestrutura, condições de trabalho e suporte psicoterapêutico estão entre as principais indicações como prevenção para o esgotamento físico e emocional.
\end{abstract}

Palavras-chave: Esgotamento profissional; Síndrome de Burnout; Pessoal de Saúde; COVID-19.

\begin{abstract}
The present work carried out a survey of the prevalence of Burnout Syndrome (BS) in health professionals, who were in the frontline in the confrontation of the pandemic of COVID-19. The methodological process followed the PRISMA and COCHRANE recommendations. The phases of screening, eligibility and data extraction were performed by independent reviewers in standardized spreadsheets. After this process, 26 cross-sectional studies were included. The main outcomes found corroborate the variation of the mean scores in the three dimensions of BS according to the professional area, site and public affected. Most of the studies pointed nursing professionals as the most affected population, with higher prevalence of compatible scores in the three dimensions of SB, and the female population was up to $60 \%$ more affected than the male population. These data allow us to infer that more effective measures need to be taken regarding the mental well-being of these professionals. Measures related to infrastructure, working conditions, and psychotherapeutic support are among the main indications for preventing physical and emotional exhaustion.
\end{abstract}


Keywords: Burnout professional; Burnout Syndrome; Personnel health; Coronavirus infections.

\section{Resumen}

El presente trabajo realizó una encuesta sobre la prevalencia del Síndrome de Burnout (BS) en los profesionales de la salud, que estaban en primera línea para enfrentar la pandemia del COVID-19. El proceso metodológico siguió las recomendaciones PRISMA y COCHRANE y las etapas de cribado, elegibilidad y extracción de datos fueron realizadas por revisores independientes, en hojas de cálculo estandarizadas. Tras este proceso, se incluyeron 26 estudios transversales. Los principales resultados encontrados corroboran la variación de las puntuaciones medias en las tres dimensiones del burnout según el área profesional, el lugar y el público afectado. La mayoría de los estudios señalaron a los profesionales de enfermería como las poblaciones más afectadas y con mayor prevalencia de puntuaciones compatibles en las tres dimensiones del SB, siendo la población femenina hasta un $60 \%$ más afectada que la masculina. Estos datos permiten deducir que es necesario tomar medidas más eficaces en relación con el bienestar mental de estos profesionales. Las medidas relacionadas con la infraestructura, las condiciones de trabajo y el apoyo psicoterapéutico son algunas de las principales indicaciones para prevenir el agotamiento físico y emocional.

Palavras clave: Burnout; Síndrome de Burnout; Personal sanitário; COVID-19.

\section{Introdução}

Em janeiro de 2020, a Organização Mundial da Saúde (OMS) declarou como uma emergência de saúde pública de preocupação internacional a ocorrência de uma nova doença viral causada por um vírus da família coronavírus. Ainda em março desse ano, a OMS passou a considerar o surto pelo SARS-CoV-2 como uma pandemia (WHO, 2020). De imediato, o aumento na demanda e falta de equipamentos de proteção individual (EPI's), a transmissibilidade e letalidade do vírus, e o medo da exposição dos profissionais na linha de frente, trouxe à tona a preocupação acerca do bem-estar físico e mental desses profissionais (Moazzami et al., 2020).

Garantir o bem-estar dos trabalhadores da saúde seria de fundamental importância para enfretamento da pandemia pelo coronavírus. Entretanto, como resultado do medo generalizado em volta da pandemia, os profissionais da saúde sofreram e sofrem com uma enorme pressão na carga de trabalho, agravando questões como a depressão, ansiedade e esgotamento físico e emocional. As principais causas de sofrimento psíquico entre estes profissionais incluem longas horas de trabalho, distúrbios do sono, fadiga debilitante e o pânico de contrair o vírus e colocar sua família em risco (Moazzami et al., 2020).

Muito embora existam diversos modelos desenvolvidos para definir a SB, o modelo mais difundido é o proposto por Christina Maslach em 1978. Nele, o burnout é descrito como um conjunto de sintomas de dimensões físicas e psicológicas, que incluem atitudes e perspectivas negativas em relação ao trabalho, vida e outras pessoas, sensação de esgotamento emocional, e diminuição da competência pessoal, como resultado da exaustão, fadiga, desespero e desesperança, podendo atingir profissionais de todas as áreas e setores, mas principalmente os profissionais da saúde. De acordo com este modelo, existem três subdimensões diferentes de burnout: a exaustão emocional, a despersonalização e a sensação de redução e incapacidade de realização profissional e pessoal (Brewer \& Shapard, 2004).

Os sintomas da síndrome de burnout podem ser divididos em psíquicos e comportamentais, e são desencadeados por gatilhos diferentes a depender da função exercida, e a área ocupacional do indivíduo. Dentre os sintomas psíquicos, destacamse a falta de atenção e de concentração, alterações na memória, redução da velocidade do pensamento, sentimento de solidão, baixa autoestima e dificuldades de autoaceitação, impaciência, desanimo e astenia, normalmente associados a um episódio depressivo (Pereira, 2002). Já os sintomas comportamentais incluem irritabilidade, negligencia diante das questões ocupacionais, incapacidade de relaxamento, perda de iniciativa, dificuldade na aceitação de mudanças, agressividade dentro e fora do trabalho, consumo excessivo de substancias e bebidas como bebidas alcoólicas, café, cigarro, tranquilizantes ou estimulantes, entre outros (Koch \& Broich, 2012).

O Maslach Burnout Inventory (MBI) é aceito como o padrão ouro no rastreamento do risco e gravidade da síndrome de burnout. O MBI consiste em uma escala composta por 22 itens ou questões, cujo objetivo é determinar o desgaste físico e 
emocional, e que inclui as três dimensões do modelo proposto por Maslach para o burnout (Murat et al., 2020). Assim, ao final do questionário, quanto maior o nível de burnout, menor a pontuação de realização pessoal e maior a pontuação de exaustão emocional e despersonalização (Lange et al., 2020). Diante disso, o presente trabalho tem por objetivo realizar através de revisão sistemática um levantamento acerca da prevalência da síndrome de burnout em profissionais de saúde que atuaram e atuam no enfrentamento da pandemia.

\section{Metodologia}

\section{- Estratégia de busca:}

A primeira fase da pesquisa consistiu no levantamento bibliográfico de estudos nas plataformas de dados National Library of Medicine (NIH/PUBMED) e Scientific Electronic Library Online (SciELO), onde foram utilizados os descritores do tipo Medical Subject Headings (MeSH): síndrome de burnout (Burnout syndrome), profissionais da saúde (Health providers), e COVID-19 (Coronavirus infections). E usando operadores booleanos AND, OR e NOT para cruzar os descritores acima citados nas bases de dados mencionadas que abordassem "burnout em profissionais da saúde no enfrentamento da pandemia pelo COVID-19". A Tabela 1 descreve as estratégias de buscas utilizadas em cada uma das bases de dados e suas respectivas datas de pesquisa, número de estudos encontrados e número de artigos selecionados.

Tabela 1. Descrição da estratégia de busca adotada.

\begin{tabular}{|c|c|c|c|c|}
\hline $\begin{array}{l}\text { Base de } \\
\text { dados: }\end{array}$ & Estratégia de busca: & $\begin{array}{l}\text { Data de } \\
\text { busca: }\end{array}$ & Resultados: & Selecionados: \\
\hline SciELO & $\begin{array}{l}\text { ("Burnout, Professional" OR "Burnout") AND (“Coronavirus } \\
\text { Infections" OR "Covid-19") }\end{array}$ & $14 / 05 / 2021$ & 10 & 3 \\
\hline PubMED & $\begin{array}{l}\text { "Burnout, Psychological"[Mesh] OR Burnout [tiab] } \\
\text { "Health Personnel"[Mesh] OR "Personnel, Health"[tiab] OR "Health } \\
\text { Care Providers"[tiab] OR "Health Care Provider"[tiab] OR "Provider, } \\
\text { Health Care"[tiab] } \\
\text { "COVID-19"[Mesh] OR "COVID-19"[tiab] OR coronavirus[tiab] } \\
\text { AND "Coronavirus Infections"[Mesh] }\end{array}$ & $14 / 05 / 2021$ & 279 & 26 \\
\hline
\end{tabular}

Fonte: Autores (2021).

\section{- Critérios de seleção}

Cerca de 289 estudos e publicações foram encontrados, e a partir da leitura inicial do título e resumo pelos revisores através da ferramenta de leitura colaborativa Rayyan Inc., foram estabelecidos os critérios contidos na Tabela 2.

Tabela 2. Critérios de inclusão e exclusão dos estudos.

\begin{tabular}{c|c} 
Critérios para Inclusão: & Critérios para Exclusão: \\
\hline $\begin{array}{c}\text { Artigos publicados em inglês, português e espanhol; } \\
\text { Estudos transversais que avaliam a síndrome de burnout em } \\
\text { profissionais da saúde; }\end{array}$ & $\begin{array}{c}\text { Artigos que não avaliam a prevalência de burnout no } \\
\text { período da pandemia pela COVID-19; }\end{array}$ \\
\hline Artigos publicados entre 2019, 2020 e 2021; & $\begin{array}{c}\text { Estudos secundários e de revisão (revisões de literatura, } \\
\text { revisões sistemáticas, overview e etc.); }\end{array}$ \\
\hline Artigos cujos textos estejam disponíveis por completo; & Estudos cuja populações não eram profissionais da saúde; \\
\hline
\end{tabular}

Fonte: Autores (2021). 
A revisão e leitura para seleção dos estudos foi realizada do dia 24 de maio de 2021 a 10 de junho de 2021, tendo 125 artigos incluídos. A etapa seguinte foi a triagem, por dois revisores independentes, em que $k=0,93$ de concordância considerada perfeita. A elegibilidade se deu através da leitura integra dos 53 artigos selecionados, avaliando objetivos e metodologia como critérios, onde 26 estudos transversais que utilizaram o $M B I$ como ferramenta de rastreio do burnout foram selecionados para a etapa de extração de dados e a síntese qualitativa final.

\section{- Avaliação da qualidade metodológica dos artigos incluídos na síntese qualitativa}

Segundo a Diretriz Metodológica de Revisão Sistemáticas (2012), a qualidade da evidencia está relacionada ao grau de confiança que se deposita em determinados estudos, onde, quanto mais forte for esse estudo maior será a sua comprovação. Porém, no presente estudo de revisão não foi realizada a análise da qualidade metodológica dos artigos incluídos para síntese qualitativa, pois, não foram encontrados instrumentos validados e traduzidos que avaliem a qualidade metodológica para estudos transversais.

\section{- Financiamento e conflito de interesse}

Esse estudo foi desenvolvimento sem financiamento e não houve conflito de interesse.

\section{Resultados}

\section{- Caracterização demográfica dos estudos incluídos:}

A Figura 1 traz o fluxograma baseado nas recomendações PRISMA, que mapeou o presente estudo. A busca identificou 289 artigos, onde, 53 seguiram para a leitura completa e ao final 26 foram estudos transversais foram incluídos.

Figura 1. Fluxograma PRISMA para mapeamento da revisão sistemática.

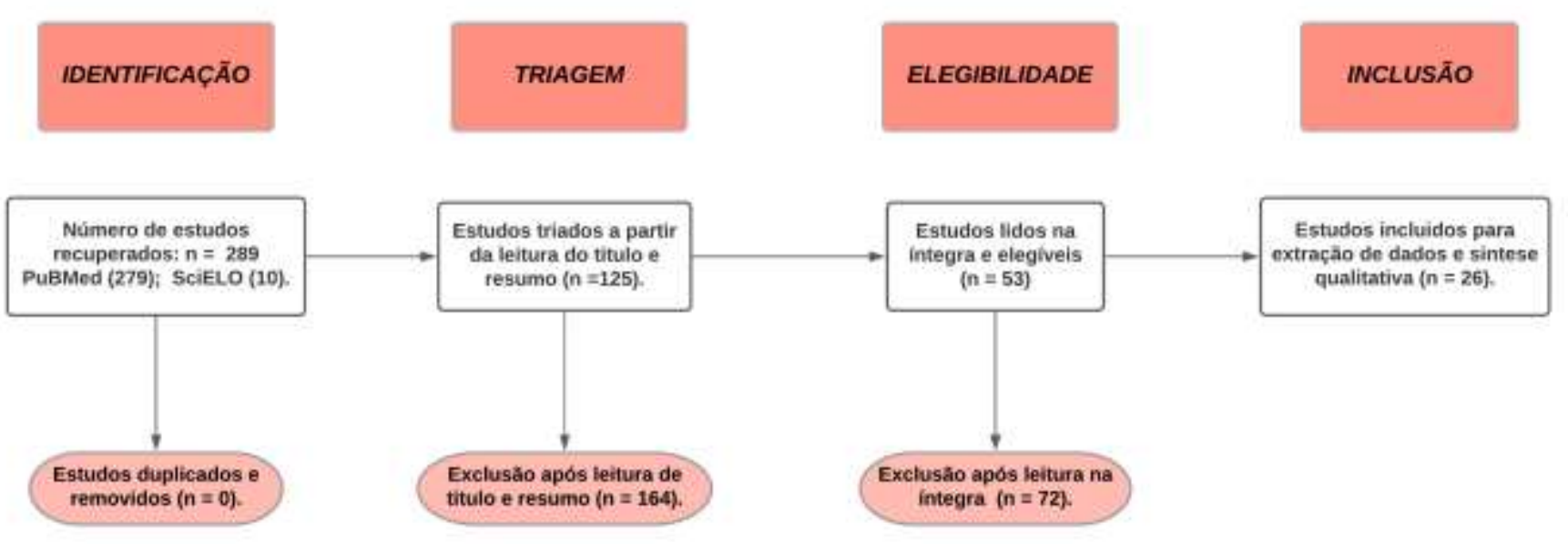

Fonte: Autores (2021).

Foram incluídos artigos da Arabia Saudita (1) (Alkhamees et al.. .), Brasil (1) (Freitas et al.. .), Canadá (2) (Khan et al. ; Lim et al.. .), China (2) (Chen et al.. .; Wu et al.. .), Coreia do Sul (1) (Park et al.. .), Egito (1) (Abdelhafiz et al.), Equador (1) (Vinueza-Veloz, et al.. .), Espanha (2) (Macía-Rodríguez et al..; Luceño-Moreno et al..), Estados Unidos (1) (Khalafallah et al..), Grécia (1) (Pappa et al..), Holanda (2) (Kok et al..; Meynaar et al..), Itália (4) (Barello et al..; Lasalvia et al..; Orrù et al..; 
Rapisarda et al..), Japão (2) (Matsuo et al..; Nishimura et al..), Malásia (1) (Tsan et al..), Polônia (1) (Pniak et al..), Portugal (1) (Zerbini et al..), Suíça (1) (Aebischer et al..), Turquia (1) (Yörük \& Güler).

Todos os 26 estudos são classificados como estudos transversais e foram conduzidos entre os anos de 2020 e 2021 , e a faixa etária dos participantes $(\mathrm{n}=23639)$ foi entre 20 (Vinueza-Veloz et al..) e 69 anos (Barello et al..). Os estudos incluídos possuíram uma média de 909 participantes, variando entre 33 (Nishimura et al..) a 12596 participantes (Chen et al..). Indivíduos do sexo feminino apresentaram uma maior participação, tendo uma variação de 50,45\% em participantes do Egito (Abdelhafiz et al..) e 95\% em participantes da China (Chen et al..), conforme mostra o Quadro 1.

\section{- Prevalência de burnout e as suas dimensões em profissionais da saúde}

Todos os 26 estudos incluídos utilizaram o MBI como instrumento psicométrico para rastreio e avaliação do burnout, que foi aplicado junto aos questionários para obtenção dos dados demográficos. Foram incluídos estudos que tinham como público os profissionais da enfermagem (4) (Chen et al..; Vinueza-Veloz et al..; Wu et al..; Yörük \& Güler), fisioterapeutas (1) (Pniak et al..), internos de medicina (3) (Aebischer et al.., Khan et al., Macía-Rodríguez et al..), médicos (3) (Lim et al..; Park et al..; Vinueza-Veloz et al..), médicos anestesiologistas e intensivistas (2) (Meynaar et al..; Tsan et al..), neurocirurgiões (1) (Khalafallah et al..). 
Research, Society and Development, v. 10, n. 16, e167101623591, 2021

(CC BY 4.0) | ISSN 2525-3409 | DOI: http://dx.doi.org/10.33448/rsd-v10i16.23591

Quadro 1. Características demográficas dos artigos incluídos na revisão.

\begin{tabular}{|c|c|c|c|c|c|c|c|c|c|c|c|}
\hline \multirow[b]{2}{*}{ Titulo } & \multirow[b]{2}{*}{ Autor } & \multirow[b]{2}{*}{ Ano } & \multirow[b]{2}{*}{ País/Local } & \multirow[b]{2}{*}{ Publico } & \multirow{2}{*}{$\begin{array}{l}\text { Participantes } \\
\text { (n) }\end{array}$} & \multirow[b]{2}{*}{ Sexo } & \multirow{2}{*}{$\begin{array}{l}\text { Idade } \\
\text { (anos) }\end{array}$} & \multicolumn{4}{|c|}{ Prevalência } \\
\hline & & & & & & & & EE & DP & $\mathbf{R P}$ & $\begin{array}{l}\text { Percentual } \\
(\%)\end{array}$ \\
\hline $\begin{array}{l}\text { Prevalence, Associated Factors, and Consequences of Burnout Among } \\
\text { Egyptian Physicians During COVID-19 Pandemic }\end{array}$ & $\begin{array}{l}\text { ABDELHAFIZ, A. S. } \\
\text { et al. }\end{array}$ & 2020 & Egito & $\begin{array}{c}\text { Profissionais da Saúde } \\
\text { em geral }\end{array}$ & 220 & $\begin{array}{l}50,45 \% \\
\text { Feminino }\end{array}$ & $28-38$ & 20,67 & 7,2 & 18,53 & $36,36 \%$ \\
\hline $\begin{array}{l}\text { Physical and psychological health of medical students involved in the } \\
\text { coronavirus disease } 2019 \text { response in Switzerland }\end{array}$ & AEBISCHER, O. et al. & 2020 & Suíça & Internos de Medicina & 788 & $\begin{array}{c}65,5 \% \\
\text { Feminino }\end{array}$ & $22-32$ & 33 & 25 & $N / R$ & $16,90 \%$ \\
\hline $\begin{array}{l}\text { Burnout and depression among psychiatry residents during COVID-19 } \\
\text { pandemic }\end{array}$ & $\begin{array}{l}\text { ALKHAMEES, A. A. } \\
\text { et al. }\end{array}$ & 2021 & $\begin{array}{l}\text { Arábia } \\
\text { Saudita }\end{array}$ & $\begin{array}{l}\text { Residentes de } \\
\text { Psiquiatria }\end{array}$ & 121 & $\begin{array}{c}57,9 \% \\
\text { Feminino }\end{array}$ & $24-33$ & 26,4 & 10,7 & 24 & $27,30 \%$ \\
\hline $\begin{array}{l}\text { Burnout and somatic symptoms among frontline healthcare } \\
\text { professionals at the peak of the Italian COVID-19 pandemic }\end{array}$ & BARELLO, S. et al. & 2020 & Itália & $\begin{array}{c}\text { Profissionais da Saúde } \\
\text { em Geral }\end{array}$ & 1153 & $\begin{array}{l}95 \% \\
\text { Feminino }\end{array}$ & $23-69$ & 27,8 & 6,1 & 37,5 & $N / R$ \\
\hline $\begin{array}{l}\text { A Large-Scale Survey on Trauma, Burnout, and Posttraumatic Growth } \\
\text { among Nurses during the COVID-19 Pandemic }\end{array}$ & CHEN, R. et al. & 2021 & China & Enfermeiros & 12596 & $\begin{array}{c}84,5 \% \\
\text { Feminino } \\
\end{array}$ & $25-33$ & 19,1 & 5,5 & 19 & $N / R$ \\
\hline $\begin{array}{l}\text { Preditores da síndrome de Burnout em técnicos de enfermagem de } \\
\text { unidade de terapia intensiva durante a pandemia da COVID-19 }\end{array}$ & FREITAS, R. F. et al. & 2021 & Brasil & $\begin{array}{l}\text { Técnicos de } \\
\text { enfermagem }\end{array}$ & 94 & $\begin{array}{l}88,7 \% \\
\text { Masculino }\end{array}$ & $27-45$ & 26,7 & 24 & 11,5 & $25,50 \%$ \\
\hline $\begin{array}{l}\text { Burnout and career satisfaction among attending neurosurgeons during } \\
\text { the COVID-19 pandemic }\end{array}$ & $\begin{array}{l}\text { KHALAFALLAH, A. } \\
\text { M. et al. }\end{array}$ & 2020 & $\begin{array}{l}\text { Estados } \\
\text { Unidos }\end{array}$ & Neurocirurgiões & 407 & $\begin{array}{c}51 \% \\
\text { Masculino }\end{array}$ & $30-60$ & 7,05 & 2,73 & 15,06 & $20,40 \%$ \\
\hline $\begin{array}{l}\text { Cross-sectional survey on physician burnout during the COVID-19 } \\
\text { pandemic in Vancouver, Canada: the role of gender, ethnicity and } \\
\text { sexual orientation }\end{array}$ & KHAN, N. et al. & 2021 & Canadá & Internos de Medicina & 302 & $\begin{array}{l}79,0 \% \\
\text { Feminino }\end{array}$ & $25-65$ & $N / R$ & $N / R$ & $N / R$ & $68 \%$ \\
\hline $\begin{array}{l}\text { Coronavirus Disease } 2019 \text { Immediately Increases Burnout Symptoms } \\
\text { in ICU Professionals: A Longitudinal Cohort Study }\end{array}$ & KOK, N. et al. & 2021 & Holanda & $\begin{array}{c}\text { Profissionais da Saúde } \\
\text { em geral }\end{array}$ & 233 & $\begin{array}{c}79,0 \% \\
\text { Feminino }\end{array}$ & $32-51$ & 1,57 & 1,1 & 4,24 & $36 \%$ \\
\hline $\begin{array}{l}\text { Emergency medicine physician burnout and wellness in Canada before } \\
\text { COVID19: A national survey }\end{array}$ & LIM, R. et al. & 2020 & Canadá & $\begin{array}{l}\text { Profissionais da Saúde } \\
\text { em geral }\end{array}$ & 384 & $\begin{array}{c}74,8 \\
\text { Feminino }\end{array}$ & $31-50$ & 20,05 & 14,55 & 32,17 & $N / R$ \\
\hline $\begin{array}{l}\text { Symptoms of Posttraumatic Stress, Anxiety, Depression, Levels of } \\
\text { Resilience and Burnout in Spanish Health Personnel during the } \\
\text { COVID-19 Pandemic }\end{array}$ & $\begin{array}{l}\text { LUCEÑO-MORENO, } \\
\text { L. et al. }\end{array}$ & 2020 & Espanha & Médicos & 1422 & $\begin{array}{c}55,6 \% \\
\text { Masculino }\end{array}$ & $32-54$ & 34,07 & 16,35 & 45,64 & $33,00 \%$ \\
\hline $\begin{array}{l}\text { Burn-out syndrome in Spanish internists during the COVID-19 } \\
\text { outbreak and associated factors: a cross-sectional survey }\end{array}$ & $\begin{array}{c}\text { MACÍA- } \\
\text { RODRÍGUEZ, C. et al. }\end{array}$ & 2021 & Espanha & $\begin{array}{c}\text { Profissionais da Saúde } \\
\text { em geral }\end{array}$ & 1015 & $\begin{array}{c}86,4 \% \\
\text { Feminino }\end{array}$ & $31-47$ & $N / R$ & $N / R$ & $N / R$ & $41,10 \%$ \\
\hline $\begin{array}{l}\text { Prevalence of Health Care Worker Burnout During the Coronavirus } \\
\text { Disease } 2019 \text { (COVID-19) Pandemic in Japan }\end{array}$ & MATSUO, T. et al. & 2020 & Japão & Internos de Medicina & 312 & $\begin{array}{c}62,9 \% \\
\text { Feminino }\end{array}$ & $25-38$ & $N / R$ & $N / R$ & $N / R$ & $58,30 \%$ \\
\hline $\begin{array}{l}\text { Burnout, resilience and work engagement among Dutch intensivists in } \\
\text { the aftermath of the COVID-19 crisis: A nationwide survey }\end{array}$ & MEYNAAR, I. A. et al. & 2021 & Holanda & $\begin{array}{c}\text { Profissionais da Saúde } \\
\text { em geral }\end{array}$ & 162 & $\begin{array}{l}71,5 \% \\
\text { Feminino }\end{array}$ & $36-46$ & $N / R$ & $N / R$ & $N / R$ & $31,40 \%$ \\
\hline $\begin{array}{l}\text { Burnout of Healthcare Workers amid the COVID-19 Pandemic: A } \\
\text { Japanese Cross-Sectional Survey }\end{array}$ & NISHIMURA, Y. et al. & 2021 & Japão & Intensivistas & 33 & $\begin{array}{c}60,5 \% \\
\text { Masculino }\end{array}$ & $N / R$ & $N / R$ & $N / R$ & $N / R$ & $16,70 \%$ \\
\hline $\begin{array}{l}\text { Secondary Traumatic Stress and Burnout in Healthcare Workers during } \\
\text { COVID-19 Outbreak }\end{array}$ & ORRÙ, G. et al. & 2021 & Itália & $\begin{array}{c}\text { Profissionais da Saúde } \\
\text { em Geral }\end{array}$ & 184 & $\begin{array}{c}72,7 \% \\
\text { Feminino }\end{array}$ & $35-57$ & 24,8 & 6,6 & 27,4 & $50 \%$ \\
\hline
\end{tabular}


Research, Society and Development, v. 10, n. 16, e167101623591, 2021

(CC BY 4.0) | ISSN 2525-3409 | DOI: http://dx.doi.org/10.33448/rsd-v10i16.23591

\begin{tabular}{|c|c|c|c|c|c|c|c|c|c|c|c|}
\hline $\begin{array}{l}\text { From Recession to Depression? Prevalence and Correlates of } \\
\text { Depression, Anxiety, Traumatic Stress and Burnout in Healthcare } \\
\text { Workers during the COVID-19 Pandemic in Greece: A Multi-Center, } \\
\text { Cross-Sectional Study }\end{array}$ & PAPPA, S. et al. & 2021 & Grécia & $\begin{array}{l}\text { Profissionais da saúde } \\
\text { em geral }\end{array}$ & 464 & $\begin{array}{c}50,5 \% \\
\text { Masculino }\end{array}$ & $31-51$ & 19,66 & 7,53 & 32,48 & $\mathrm{~N} / \mathrm{R}$ \\
\hline $\begin{array}{l}\text { Psychological distress among infectious disease physicians during the } \\
\text { response to the COVID-19 outbreak in the Republic of Korea }\end{array}$ & PARK, S. Y. et al. & 2020 & $\begin{array}{l}\text { Coreia do } \\
\quad \text { Sul }\end{array}$ & $\begin{array}{l}\text { Profissionais da Saúde } \\
\text { em Geral }\end{array}$ & 115 & $\begin{array}{c}68 \% \\
\text { Feminino }\end{array}$ & $37-48$ & 27,67 & 36,84 & 14,25 & $\mathrm{~N} / \mathrm{R}$ \\
\hline $\begin{array}{l}\text { Occupational burnout among active physiotherapists working in } \\
\text { clinical hospitals during the COVID-19 pandemic in south-eastern } \\
\text { Poland }\end{array}$ & PNIAK, B. et al. & 2021 & Polônia & Médicos & 106 & $\begin{array}{c}58,3 \% \\
\text { Feminino }\end{array}$ & $24-39$ & 34,92 & 10,55 & 31,66 & $90,40 \%$ \\
\hline $\begin{array}{l}\text { The Early Impact of the Covid-19 Emergency on Mental Health } \\
\text { Workers: A Survey in Lombardy, Italy }\end{array}$ & RAPISARDA, F. et al. & 2020 & Itália & Fisioterapeutas & 337 & $\begin{array}{c}60 \% \\
\text { Feminino }\end{array}$ & $32-56$ & 34,07 & 18,78 & 24,54 & $N / R$ \\
\hline $\begin{array}{l}\text { To burn-out or not to burn-out: a cross-sectional study in healthcare } \\
\text { professionals in Spain during COVID-19 pandemic }\end{array}$ & TORRENTE, M. et al. & 2021 & Espanha & $\begin{array}{l}\text { Profissionais da saúde } \\
\text { em geral }\end{array}$ & 643 & $\begin{array}{c}76,8 \% \\
\text { Feminino }\end{array}$ & $31-40$ & 16,7 & 3 & 13,7 & $20 \%$ \\
\hline $\begin{array}{l}\text { A survey on burnout and depression risk among anaesthetists during } \\
\text { COVID-19: the tip of an iceberg? }\end{array}$ & TSAN, S. E. H. et al. & 2021 & Malásia & Anestesiologistas & 88 & $\begin{array}{c}54 \% \\
\text { Feminino }\end{array}$ & $28-36$ & 21,35 & 8,74 & 29,2 & $55,30 \%$ \\
\hline $\begin{array}{l}\text { Burnout syndrome among Ecuadorian medical doctors and nurses } \\
\text { during COVID-19 pandemic }\end{array}$ & $\begin{array}{l}\text { VINUEZA-VELOZ, A. } \\
\text { F. et al. }\end{array}$ & 2020 & Equador & $\begin{array}{l}\text { Médicos e } \\
\text { Enfermeiros }\end{array}$ & 224 & $\begin{array}{c}63,58 \% \\
\text { Feminino }\end{array}$ & $20-36$ & $N / R$ & $\mathrm{~N} / \mathrm{R}$ & $N / R$ & $78,15 \%$ \\
\hline $\begin{array}{l}\text { A Comparison of Burnout Frequency Among Oncology Physicians and } \\
\text { Nurses Working on the Frontline and Usual Wards During the } \\
\text { COVID-19 Epidemic in Wuhan, China }\end{array}$ & WU, Y. et al. & 2020 & China & $\begin{array}{l}\text { Enfermeiros e } \\
\text { Médicos }\end{array}$ & 190 & $\begin{array}{l}83 \% \\
\text { Feminino }\end{array}$ & $28-37$ & 27,44 & 9,3 & 34,41 & $N / R$ \\
\hline $\begin{array}{l}\text { The relationship between psychological resilience, burnout, stress, and } \\
\text { sociodemographic factors with depression in nurses and midwives } \\
\text { during the COVID-19 pandemic: A cross-sectional study in Turkey }\end{array}$ & $\begin{array}{l}\text { YÖRÜK, S. and } \\
\text { GÜLER, D. }\end{array}$ & 2021 & Turquia & Enfermeiros obstetras & 384 & $N / R$ & $28-40$ & 20,06 & 6,42 & 22,7 & $N / R$ \\
\hline $\begin{array}{l}\text { Psychosocial burden of healthcare professionals in times of COVID-19 } \\
\text { - a survey conducted at the University Hospital Augsburg }\end{array}$ & ZERBINI, G. et al. & 2020 & Portugal & $\begin{array}{c}\text { Profissionais da Saúde } \\
\text { em geral }\end{array}$ & 110 & $N / R$ & $\mathrm{~N} / \mathrm{R}$ & $N / R$ & $\mathrm{~N} / \mathrm{R}$ & $N / R$ & $55 \%$ \\
\hline
\end{tabular}

$\mathrm{EE}=$ Esgotamento Emocional; DP = Despersonalização; RP = Realização Pessoal; MBI = Maslach Burnout Inventory; N/R = Não relatado. Fonte: Autores (2021). 
profissionais da saúde em geral (incluindo enfermeiros, farmacêuticos, fisioterapeutas, médicos, técnicos e etc.) (11) (Matsuo et al..; Abdelhafiz et al..; Barello et al..; Kok et al..; Lasalvia et al..; Luceño-Moreno et al..; Nishimura et al..; Orrù et al..; Pappa et al..; Rapisarda et al..; Zerbini et al..), residentes em psiquiatria (1) (Alkhamees et al..), técnicos em enfermagem (1) (Freitas et al..).

A prevalência do burnout foi avaliada em todos os estudos por meio do questionário MBI, que afere cada uma das dimensões do burnout: Esgotamento emocional (EE), Despersonalização (DP), e Realização pessoal (RP). Oito estudos (Barello et al..; Chen et al..; Lasalvia et al.; Orrù et al..; Pappa et al..; Pniak et al..; Wu et al..; Yörük \& Güler) não relataram a prevalência geral do burnout em porcentagem, contudo, trouxeram os dados referentes a média dos escores multidimensionais do MBI.

Entre os profissionais da enfermagem, dois estudos indicaram escores moderados para exaustão emocional, despersonalização e realização pessoal (EE: 19,1 \pm 10,0; DP: 5,5 $\pm 4,6$; RP: 19,0 \pm 8,4), e que os profissionais do sexo feminino apresentaram pontuações significativamente maiores do que os do sexo masculino nas escalas de esgotamento emocional (MBI-EE, p = 0,007) (Chen, R et. al). Observou-se também uma relação significativa entre as dimensões do burnout e a depressão. Sendo uma relação negativa fraca entre depressão e realização pessoal $(r=-0,10, p<0,05)$, uma relação positiva forte entre depressão e despersonalização $(r=0,20, \mathrm{p}<0,001)$, e uma relação positiva moderada entre depressão e exaustão emocional ( $\mathrm{r}=0,49, \mathrm{p}<0,001)$ (Yörük \& Güler).

Na Polônia, um estudo feito entre fisioterapeutas que trabalharam profissionalmente durante a pandemia, maiores taxas de burnout foram observadas nos trabalhadores do sexo masculino, em comparação ao feminino, nas dimensões de esgotamento emocional (EE: Homens: 34,70 \pm 5,70 e Mulheres: 31,03 \pm 3,58), despersonalização (DP: Homens: $18,78 \pm 2,8$ e Mulheres: 14,90 \pm 2,26; e realização pessoal (RP: Homens: 24,54 \pm 3,22 e Mulheres: 27,17 $\pm 2,27$ ). Maiores taxas de burnout foram refletidas nas unidades de terapia intensiva (UTI's) e departamento de anestesiologia (EE: 40,89 \pm 5,62; DP: 21,39 \pm 3,49 e RP: 23,07 $\pm 3,03$ ), em comparação com os outros departamentos (Pniak et al..).

Em outro estudo realizado em julho de 2020 em um hospital da China, que comparou a frequência de burnout entre as equipes da enfermaria comum e a equipe da linha de frente da enfermaria COVID-19, obteve-se que o burnout é significativamente menor na equipe da enfermaria comum (13\%) do que na equipe de linha de frente da COVID-19 (39\%), sendo maior a prevalência para as dimensões de exaustão emocional e despersonalização ( $\mathrm{P}<0,0001)$. Enquanto que o baixo grau da realização pessoal é menor no grupo da linha de frente (39\%), quando comparada com a equipe da enfermaria comum $(61 \%)(\mathrm{P}=0,0002)$ (Wu et al.).

Na Itália, em agosto de 2020, Barello S. et.al., realizou um estudo onde houve uma correlação significativa entre o sexo $(\mathrm{p}<0,001)$ e profissão $(\mathrm{p}<0,006)$ nas frequências das dimensões do burnout, com profissionais médicos masculinos apresentando esgotamento emocional, despersonalização e baixo grau de realização pessoal com menos frequência do que profissionais da enfermagem do sexo feminino. Além disso, na comparação entre os profissionais de saúde que trabalharam em países que adotaram políticas de lockdown rígidas $(\mathrm{n}=96)$ e países com políticas de lockdown mais suaves $(\mathrm{n}=80)$, obteve-se pontuações mais altas nos escores de despersonalização (MBI-DP: p = 0,033) e baixo grau de realização pessoal (MBI-RP: $p=$ 0,019), e as profissionais de saúde do sexo feminino apresentaram pontuações significativamente maiores do que os do sexo masculino na escala de exaustão emocional (MBI-EE: p = 0,007) (Orrù et al..).

Segundo Lasalvia et al.., no geral, 38,3\% dos participantes apresentaram sintomas de grande exaustão emocional, 46,5\% apresentaram alto grau de despersonalização, e 26,5\% apresentaram baixo grau de realização pessoal de baixa eficácia profissional, sendo em geral, mais prevalente entre os funcionários que trabalham nas UTIs comuns e de cuidados subintensivos da COVID. Enquanto que na Grécia, Pappa et al.., relata altos níveis de burnout nos profissionais da linha de 
frente em todas as três dimensões: exaustão emocional foi alta em 44,01\% dos participantes, despersonalização foi alta em $92,22 \%$, e 49,34 \% para baixo grau de realização pessoal entre os participantes do estudo.

Outros dezoitos estudos (Abdelhafiz et al.., Aebischer et al.., Alkhamees et al.., Freitas et al.., Khalafallah et al.., Khan et al.., Kok et al.., Lim et al.., Luceño-Moreno et al.., Macía-Rodríguez et al.., Matsuo et al.., Meynaar et al.., Nishimura et al.., Park et al.., Rapisarda et al.., Tsan et al.., Vinueza-Veloz et al.., Zerbini et al..), incluíram a prevalência em percentual geral de burnout em pelo menos uma das dimensões entre os participantes.

Dez estudos (Abdelhafiz et al., Aebischer et al., Alkhamees et al., Freitas et al., Khalafallah et al., Kok et al., Lim et al.., Luceño-Moreno et al., Matsuo et al., Meynaar et al., Rapisarda et al.), trazem que a prevalência de burnout foi inferior a cinquenta por cento entres os profissionais incluídos nos estudos. Nos profissionais da saúde em geral, um estudo realizado na Itália, mostrou que aproximadamente dois terços dos participantes trabalhavam em serviços ambulatoriais, sendo a maioria do sexo feminino (76,8\%), com média de idade de 44 anos e vinte por cento dos participantes obtiveram pontuação severa em pelo menos uma das dimensões de burnout (Rapisarda et al.). Em um hospital terciário em Tóquio, a prevalência geral de burnout foi de $31,4 \%$ entre os profissionais $(n=312)$. Sendo $46,8 \%$ em enfermeiros $(n=59), 36,4 \%$ em técnicos de radiologia $(\mathrm{n}=8)$, e 36,8\% em farmacêuticos ( $\mathrm{n}=7$ ) (Matsuo et al.).

Abdelhafiz et al., em um estudo realizado no Egito, apontou que A frequência de burnout foi de 36,36\% entre o grupo de profissionais participantes e que o desenvolvimento dos sintomas aumentou duas vezes diante da falta dos equipamentos de proteção individual (EPI). Outra variável relacionada positiva e significativamente com o burnout, foi a preocupação e o medo de infectar familiares e pessoas da residência, além disso obteve-se uma prevalência geral de 41,10\% entre os profissionais que atuaram na linha de frente na Espanha (Luceño-Moreno et al.). Além disso, a percepção da conduta insegura entre colegas aumenta as chances dos sintomas de burnout na análise multivariável, sendo prevalente em $36 \%$ dos profissionais participantes que apresentam agravamento em pelo menos uma das dimensões (Kok, N. et al.).

Resultados semelhantes foram encontrados por Meynaar, et al., Lim, R. et al., Khalafallah, et al., Alkhamees, et al., Aebischer, et al., em estudos realizados com médicos intensivistas, neurocirurgiões, clínicos gerais, residentes de psiquiatria e internos de medicina, respectivamente. A prevalência geral entre os profissionais médicos participantes desses estudos foi de $23,30 \%( \pm 0,065)$. Os principais fatores relatados para esse evento incluíram trabalhar em um ambiente de trabalho hostil ou difícil ( $\mathrm{p}<0,001$ ) (Khalafallah et al); a faixa etária, onde médicos com idades entre 30-39 têm quase quatro vezes mais probabilidade de sofrer com burnout (IC: 95\%) (LIM, R. et al.). Além disso houve uma relação entre burnout e sintomas depressivos, em que os entrevistados que sofreram de burnout foram significativamente mais propensos a ter sintomas depressivos (IC 95\% p <0,001) (Alkhamees et al).

Percentuais de burnout acima dos 50\% entre os participantes foram encontrados em sete estudos. Obteve-se que 90,4\% dos clínicos gerais respondentes tiveram triagem positiva para burnout (Park et al.), $78,15 \%$ entre médicos e enfermeiros com relação significativa entre idade e burnout ( $\mathrm{p}=0.04$ ) (Vinueza-Veloz et al.), aproximadamente 63,15\% entre os internos de medicina (Khan, N. et al., Macía-Rodríguez et al.), 55,3\% entre médicos anestesiologistas (Tsan et al.), 55\%, e 50\% entre profissionais da saúde em geral, respectivamente (Nishimura et al., Zerbini et al.).

\section{Discussão}

Esta revisão sistemática trouxe dados atualizados sobre a prevalência da síndrome de burnout nos profissionais da saúde durante a pandemia pela COVID-19. Foram sintetizados os resultados dos 26 artigos e mostraram que os profissionais de saúde em todo mundo apresentaram altos níveis de burnout, associados principalmente com a ansiedade e depressão, tanto durante quanto após os picos pandêmicos. Foram identificados alguns fatores de risco para essas condições, incluindo fatores sociodemográficos, como idade e sexo, e fatores sociais, como falta de apoio social, rejeição, isolamento e estigmatização dos 
transtornos mentais.

Conforme obtido em dezoito dos estudos incluídos (Aebischer et al.; Barello et al.; Chen et al.; Freitas et al.; Kok et al.; Lasalvia et al.; Luceño-Moreno et al.; Macía-Rodríguez et al.; Matsuo et al.; Nishimura et al.; Orrù et al.; Pappa et al.; Park et al.; Pniak et al.; Rapisarda et al.; Tsan et al.; Vinueza-Veloz et al.; Wu et al.), a prevalência maior foi entre os profissionais do sexo feminino, como já previsto em outros estudos. A prevalência do burnout particularmente alta em profissionais do sexo feminino se revela especialmente entre aqueles que trabalham longas horas (plantões) com pacientes internos, característica frequentemente associada entre burnout e profissionais do sexo feminino, já alto em tempos normais (Pablo, et al., 2020). Tal fato é comumente relatado e associado a frequência de sono insatisfatório durante a pandemia pela COVID-19 (Xu, et al., 2021).

Historicamente, a associação entre epidemias virais e transtornos mentais é comum. Durante o período entre os anos de 2009 e 2011, que correspondem a pandemia pelo vírus influenza (H1N1), Matsuishi et al. (2012), mostrou que profissionais em ambientes de trabalho considerados de alto risco (unidades de terapia intensiva, urgência e emergência) apresentaram maiores escores de exaustão emocional e despersonalização quando comparados com trabalhadores em ambientes considerados de trabalho de baixo risco (unidades de atenção básica). Além disso, o escore total de burnout entre os profissionais da enfermagem foi maior do que os escores dos médicos e os profissionais na faixa etária dos 40 a 50 anos sentiram maior exaustão emocional em comparação com a faixa dos 20 aos 30 anos de idade.

Durante a epidemia pelo coronavírus da síndrome respiratória do Oriente Médio (MERS-CoV) em 2015, o burnout foi maior diagnosticado nos profissionais que cuidaram de pacientes infectados ou suspeitos de MERS-CoV, quando comparados com aqueles que não o fizeram. O estresse no trabalho foi o maior fator de influência do burnout, a falta de recursos nos hospitais para o tratamento de MERS-CoV e o apoio insuficiente da família e amigos agravaram ainda mais o quadro, constata Chigwedere, et al (2021), em sua revisão sistemática.

Os estudos realizados antes pandemia pela COVID-19 já traziam dados congruentes em relação a prevalência do burnout nos profissionais da saúde, mesmo em situação fora de períodos epidêmicos. Em um estudo realizado com profissionais de unidades de cuidados intensivos, Arrogante e Aparicio-Zaldivar (2017), observaram que a maior porcentagem de profissionais afetados em alguma das três subdimensões do burnout eram indivíduos do sexo feminino, com idades entre 23 e 50 anos, e que existe uma correlação significativamente importante entre o esgotamento físico, o burnout e demais transtornos mentais, como a ansiedade. Em outro estudo feito com profissionais de hospitais públicos e privados da Africa, Khamisa, et al. (2015), mostrou uma forte associação entre os altos escores de exaustão emocional e despersonalização com a ansiedade, insônia, disfunção social, embotamento afetivo, e sintomas depressivos que variam de moderados a graves (p $<0,05)$.

Os fatores ocupacionais de risco mais citados nos estudos dessa revisão implicaram trabalhar em um ambiente de alto risco (equipes da linha de frente), funções ocupacionais específicas (por exemplo, profissionais da enfermagem) e pouca preparação e experiência de trabalho. No contexto de pandemia, e como visto anteriormente em outras epidemias, a saúde mental dos profissionais da saúde sempre entra em questão, e com a demanda no aumento das cargas horarias, redução das equipes e a morte de muitos profissionais no enfrentamento da COVID-19, o burnout foi um dos problemas mentais mais relatados e diagnosticados na pandemia (Shanafelt; Ripp \& Trockel, 2020).

Fatores de risco não modificáveis, como os profissionais que atuam na linha de frente (Abdelhafiz et al., Aebischer et al., Barello et al., Chen et al., Freitas et al., Khan et al., Kok et al., Lim et al., Luceño-Moreno et al., Matsuo et al., Meynaar et al., Nishimura et al.) aumentam significativamente as chances de desenvolver sintomas clínicos da síndrome de burnout. Tais dados corroboram com o que foi encontrado por Fernandez et al. (2020), que em sua revisão mostrou maior prevalência de burnout em profissionais da enfermagem, e muito embora houvesse um profundo desejo de continuar a prestar cuidados, tais 
virtudes não impediu os profissionais de nutrir medos e preocupações sobre a segurança de si mesmas e de suas famílias, exaustão física e emocional diante do aumento no número de pacientes e óbitos diários, apesar de continuarem nos esforços para salvar o máximo de vidas possíveis.

Enquanto os fatores modificáveis ocupacionais, como falta de treinamento e experiência no trabalho em pandemias virais (Khalafallah et al, Khan et al.), falta de EPI's (Macía-Rodríguez et al.), e infraestrutura inadequada por parte das organizações (Torrente et al., Tsan et al.), indicaram a necessidade de atenção em pontos estratégicos para melhoria e garantia de recursos adequados e viabilidade desses profissionais no seu campo de atuação, reduzindo assim o risco de desencadear estresse e demais sintomas do burnout. $\mathrm{O}$ apoio dos sistemas de saúde é fundamental, principalmente no período de adaptação em resposta a COVID-19, devendo ser direcionado a todos os níveis operacionais da rede, facilitando a coordenação de diferentes organizações do sistema de saúde e apoiando sempre o a capacitação e compartilhamento de informações em redes e organizações de saúde (Turner, et al., 2021).

Além da melhoria de infraestrutura e eliminação de fatores de risco modificáveis, o fornecimento de uma rede de apoio psicossocial e acompanhamento psicoterapêutico ainda é a medida mais citada (Chen et al., Luceño-Moreno et al., Pniak, et al., Tsan et al., Pappa et al.) e de maior eficácia, tanto para o manejo dos profissionais já afetados com o burnout quanto para prevenção de outros transtornos e sintomas, como a ansiedade, depressão e transtorno de estresse pós traumático (Civantos et al.). Uma revisão sistemática que buscou avaliar a redução dos escores de burnout por meio de intervenções a nível estrutural e individual, West, et al. (2016), mostrou entre os estudos incluídos que intervenções estruturais, que incluíram redução nas horas de serviço, mudanças na prática de atendimento, e treinamentos em grupos, e intervenções focadas no indivíduo, que incluíram o gerenciamento de estresse, treinamento de autocuidado, e capacitações para desenvolvimento de habilidades de comunicação, reduziram de forma consistente os índices e escores gerais de burnout entre os profissionais da saúde.

Contudo, o apoio psicológico deve ser disponibilizado não apenas para os profissionais de saúde que cuidam diretamente de pacientes com COVID-19, mas também para aqueles que estão expostos ao vírus de outras formas (por exemplo, laboratórios, serviços ambientais, e etc.), bem como para a equipe médica e não medica que compõe os demais departamentos, ressalta Busch et al. (2021) em seu estudo de revisão.

\section{Considerações Finais}

Essa revisão buscou contribuir para a crescente literatura acerca da compreensão da síndrome de burnout em profissionais de saúde e as condições associadas durante a pandemia de COVID-19. Através da análise feita nos estudos, foi possível confirmar o aumento da prevalência e a intensidade dos sintomas de burnout em profissionais de saúde durante a pandemia, sendo em geral, mais prevalente entre os profissionais da enfermagem do sexo feminino. Esse aumento gera grande preocupação, tendo em vista que tais profissionais merecem atenção e cuidado redobrado por cuidarem da saúde da população em geral.

Através da síntese qualitativa dos estudos infere-se que o burnout pode estar relacionado a uma série de fatores, como: idade, profissão, sexo, país, e principalmente a realização pessoal, condições de trabalho e reconhecimento profissional. Os pontos fortes dessa revisão incluíram o uso do instrumento MBI padronizado entre os estudos transversais incluídos. Além disso, o potencial viés foi reduzido por meio do envolvimento de mais de um revisor na avaliação da qualidade e triagem dos estudos. Contudo, apesar da rigidez com que esta revisão foi conduzida, algumas limitações precisam ser reconhecidas. Uma delas são as possíveis publicações não indexadas nas bases de dados e poderiam ter sido omitidas na etapa de busca.

Além disso, houveram poucos estudos realizados no Brasil acerca da prevalência do burnout em profissionais da saúde. Diante disso, medidas para a melhoria na infraestrutura, melhores condições de trabalho, acompanhamento psicológico juntamente com ações apropriadas para monitorar e prevenir o burnout, além da criação de programas de apoio para a gestão e 
controle dos transtornos mentais em crises sanitárias, são as principais medidas de intervenção sinalizadas para esse momento. Para o futuro, cabe o incentivo para o desenvolvimento de estudos robustos e de qualidade sobre o tema, que apesar da relevância, os estudos ainda são escassos, principalmente no Brasil.

\section{Referências}

Abdelhafiz, A. S., Ali, A., Ziady, H. H., Maaly, A. M., Alorabi, M., \& Sultan, E. A. (2020). Prevalence, associated factors, and consequences of burnout among egyptian physicians during COVID-19 pandemic. Frontiers in public health, 8, 864.

Aebischer, O., Weilenmann, S., Gachoud, D., Man, M., \& Spiller, T. R. (2020). Physical and psychological health of medical students involved in the coronavirus disease 2019 response in Switzerland. Swiss medical weekly, 150, w20418.

Al-Alawi, M., Al-Sinawi, H., Al-Qubtan, A., Al-Lawati, J., Al-Habsi, A., Al-Shuraiqi, M., et. al. (2019). Prevalence and determinants of burnout Syndrome and Depression among medical students at Sultan Qaboos University: A cross-sectional analytical study from Oman. Archives of environmental \& occupational health, 74(3), 130-139.

Alkhamees, A. A., Alaqil, N. S., Alsoghayer, A. S., \& Alharbi, B. A. (2020). Prevalence and determinants of burnout syndrome and depression among medical students at Qassim University, Saudi Arabia. Saudi Medical Journal, 41(12), 1375.

Arrogante, O., \& Aparicio-Zaldivar, E. (2017). Burnout and health among critical care professionals: The mediational role of resilience. Intensive and Critical Care Nursing, 42, 110-115.

Barello, S., Palamenghi, L., \& Graffigna, G. (2020). Burnout and somatic symptoms among frontline healthcare professionals at the peak of the Italian COVID-19 pandemic. Psychiatry research, 290, 113129

Brewer, E. W., \& Shapard, L. (2004). Employee burnout: A meta-analysis of the relationship between age or years of experience. Human resource development review, 3(2), 102-123.

Busch, I. M., Moretti, F., Mazzi, M., Wu, A. W., \& Rimondini, M. (2021). What we have learned from two decades of epidemics and pandemics: a systematic review and meta-analysis of the psychological burden of frontline healthcare workers. Psychotherapy and psychosomatics, 1-13.

Chen, R., Sun, C., Chen, J. J., Jen, H. J., Kang, X. L., Kao, C. C., \& Chou, K. R. (2021). A large-scale survey on trauma, burnout, and posttraumatic growth among nurses during the COVID-19 pandemic. International journal of mental health nursing, 30(1), 102-116.

Chigwedere, O. C., Sadath, A., Kabir, Z., \& Arensman, E. (2021). The Impact of Epidemics and Pandemics on the Mental Health of Healthcare Workers: A Systematic Review. International Journal of Environmental Research and Public Health, 18(13), 6695.

Civantos, A. M., Bertelli, A., Gonçalves, A., Getzen, E., Chang, C., Long, Q., \& Rajasekaran, K. (2020). Mental health among head and neck surgeons in Brazil during the COVID-19 pandemic: a national study. American journal of otolaryngology, 41(6), 102694.

da Silva Schuster, M., da Veiga Dias, V., \& Battistella, L. F. Maslach Burnout Inventory-General Survey (MBI-GS): Aplicação em Universidade Público Federal. Revista da Faculdade de Administração e Economia, 6(2), 182-195.

de Pablo, G. S., Vaquerizo-Serrano, J., Catalan, A., Arango, C., Moreno, C., Ferre, F., ... \& Fusar-Poli, P. (2020). Impact of coronavirus syndromes on physical and mental health of health care workers: Systematic review and meta-analysis. Journal of affective disorders, $275,48-57$.

Fernandez, R., Lord, H., Halcomb, E., Moxham, L., Middleton, R., Alananzeh, I., \& Ellwood, L. (2020). Implications for COVID-19: a systematic review of nurses' experiences of working in acute care hospital settings during a respiratory pandemic. International journal of nursing studies, $111,103637$.

Freitas, R. F., Barros, I. M. D., Miranda, M. A. F., Freitas, T. F., Rocha, J. S. B., \& Lessa, A. D. C. (2021). Preditores da síndrome de Burnout em técnicos de enfermagem de unidade de terapia intensiva durante a pandemia da COVID-19. Jornal Brasileiro de Psiquiatria, 70, 12-20.

Khalafallah, A. M., Lam, S., Gami, A., Dornbos III, D. L., Sivakumar, W., Johnson, J. N., \& Mukherjee, D. (2020). Burnout and career satisfaction among attending neurosurgeons during the COVID-19 pandemic. Clinical neurology and neurosurgery, 198, 106193.

Khamisa, N., Oldenburg, B., Peltzer, K., \& Ilic, D. (2015). Work related stress, burnout, job satisfaction and general health of nurses. International journal of environmental research and public health, 12(1), 652-666.

Khan, N., Palepu, A., Dodek, P., Salmon, A., Leitch, H., Ruzycki, S., ... \& Lacaille, D. (2021). Cross-sectional survey on physician burnout during the COVID-19 pandemic in Vancouver, Canada: the role of gender, ethnicity and sexual orientation. BMJ open, 11(5), e050380.

Koch, U., \& Broich, K. (2012). Das Burn-out-Syndrom.

Kok, N., van Gurp, J., Teerenstra, S., van der Hoeven, H., Fuchs, M., Hoedemaekers, C., \& Zegers, M. (2021). Coronavirus disease 2019 immediately increases burnout symptoms in ICU professionals: a longitudinal cohort study. Critical care medicine, 49(3), 419-427.

Lange, M., Joo, S., Couette, P. A., De Jaegher, S., Joly, F., \& Humbert, X. (2020, November). Impact on mental health of the COVID-19 outbreak among community pharmacists during the sanitary lockdown period. In Annales pharmaceutiques francaises (Vol. 78, No. 6, pp. 459-463). Elsevier Masson.

Lim, R., Van Aarsen, K., Gray, S., Rang, L., Fitzpatrick, J., \& Fischer, L. (2020). Emergency medicine physician burnout and wellness in Canada before COVID19: a national survey. Canadian Journal of Emergency Medicine, 22(5), 603-607. 
Luceño-Moreno, L., Talavera-Velasco, B., García-Albuerne, Y., \& Martín-García, J. (2020). Symptoms of posttraumatic stress, anxiety, depression, levels of resilience and burnout in Spanish health personnel during the COVID-19 pandemic. International journal of environmental research and public health, 17(15), 5514.

Macía-Rodríguez, C., de Oña, Á. A., Martín-Iglesias, D., Barrera-López, L., Pérez-Sanz, M. T., Moreno-Diaz, J., \& González-Munera, A. (2021). Burn-out syndrome in Spanish internists during the COVID-19 outbreak and associated factors: a cross-sectional survey. BMJ open, 11(2), e042966.

Matsuishi, K., Kawazoe, A., Imai, H., Ito, A., Mouri, K., Kitamura, N., ... \& Mita, T. (2012). Psychological impact of the pandemic (H1N1) 2009 on general hospital workers in Kobe. Psychiatry and clinical neurosciences, 66(4), 353-360.

Meynaar, I. A., Ottens, T., Zegers, M., van Mol, M. M., \& Van Der Horst, I. C. (2021). Burnout, resilience and work engagement among Dutch intensivists in the aftermath of the COVID-19 crisis: A nationwide survey. Journal of Critical Care, 62, 1-5.

Moazzami, B., Razavi-Khorasani, N., Moghadam, A. D., Farokhi, E., \& Rezaei, N. (2020). COVID-19 and telemedicine: Immediate action required for maintaining healthcare providers well-being. Journal of Clinical Virology, 126, 104345.

Morgantini, L. A., Naha, U., Wang, H., Francavilla, S., Acar, Ö., Flores, J. M., ... \& Weine, S. M. (2020). Factors contributing to healthcare professional burnout during the COVID-19 pandemic: a rapid turnaround global survey. PloS one, 15(9), e0238217.

Murat, M., Köse, S., \& Savaşer, S. (2021). Determination of stress, depression and burnout levels of front-line nurses during the COVID-19 pandemic. International Journal of Mental Health Nursing, 30(2), 533-543.

Nishimura, Y., Miyoshi, T., Hagiya, H., Kosaki, Y., \& Otsuka, F. (2021). Burnout of healthcare workers amid the covid-19 pandemic: A Japanese crosssectional survey. International journal of environmental research and public health, 18(5), 2434.

Organização Mundial de Saúde. (2020). Síndrome de burnout é detalhada em classificação internacional da OMS. Recuperado em 16 novembro, 2021 , de https://brasil.un.org/pt-br/83269-sindrome-de-burnout-e-detalhada-em-classificacao-internacional-da-oms/.

Organização Mundial de Saúde. (2021). Histórico da pandemia da COVID-19. Recuperado em 16 novembro, 2021, de https://www.paho.org/pt/covid19/historico-da-pandemia-covid-19.

Orrù, G., Marzetti, F., Conversano, C., Vagheggini, G., Miccoli, M., Ciacchini, R., ... \& Gemignani, A. (2021). Secondary traumatic stress and burnout in healthcare workers during COVID-19 outbreak. International journal of environmental research and public health, 18(1), 337.

Pappa, S., Athanasiou, N., Sakkas, N., Patrinos, S., Sakka, E., Barmparessou, Z., ... \& Katsaounou, P. (2021). From recession to depression? prevalence and correlates of depression, anxiety, traumatic stress and burnout in healthcare workers during the COVID-19 pandemic in Greece: A multi-center, cross-sectional study. International journal of environmental research and public health, 18(5), 2390.

Park, S. Y., Kim, B., Jung, D. S., Jung, S. I., Oh, W. S., Kim, S. W., ... \& Chang, H. H. (2020). Psychological distress among infectious disease physicians during the response to the COVID-19 outbreak in the Republic of Korea. BMC public health, 20(1), 1-7.

Patel, R. S., Bachu, R., Adikey, A., Malik, M., \& Shah, M. (2018). Factors related to physician burnout and its consequences: a review. Behavioral sciences, $8(11), 98$

Pereira, A. M. T. B. (2002). Burnout: Quando O Trabalho Ameaca O Bem. Casa do psicólogo.

Pniak, B., Leszczak, J., Adamczyk, M., Rusek, W., Matłosz, P., \& Guzik, A. (2021). Occupational burnout among active physiotherapists working in clinical hospitals during the COVID-19 pandemic in south-eastern Poland. Work, (Preprint), 1-11.

Rapisarda, F., Vallarino, M., Cavallini, E., Barbato, A., Brousseau-Paradis, C., De Benedictis, L., \& Lesage, A. (2020). The early impact of the Covid-19 emergency on mental health workers: a survey in Lombardy, Italy. International journal of environmental research and public health, $17(22), 8615$.

Schutte, N., Toppinen, S., Kalimo, R., \& Schaufeli, W. (2000). The factorial validity of the Maslach Burnout Inventory-General Survey (MBI-GS) across occupational groups and nations. Journal of Occupational and Organizational psychology, 73(1), 53-66.

Shanafelt, T., Ripp, J., \& Trockel, M. (2020). Understanding and addressing sources of anxiety among health care professionals during the COVID-19 pandemic. Jama, 323(21), 2133-2134.

Torrente, M., Sousa, P. A., Sánchez-Ramos, A., Pimentao, J., Royuela, A., Franco, F., ... \& Provencio, M. (2021). To burn-out or not to burn-out: a crosssectional study in healthcare professionals in Spain during COVID-19 pandemic. BMJ open, 11(2), e044945.

Tsan, S. E. H., Kamalanathan, A., Lee, C. K., Zakaria, S. A., \& Wang, C. Y. (2020). A survey on burnout and depression risk among anaesthetists during COVID-19: the tip of an iceberg?. Anaesthesia.

Turner, S., Botero-Tovar, N., Herrera, M. A., Kuhlmann, J. P. B., Ortiz, F., Ramírez, J. C., \& Maldonado, L. F. (2021). Systematic review of experiences and perceptions of key actors and organisations at multiple levels within health systems internationally in responding to COVID-19. Implementation Science, $16(1), 1-13$.

Vinueza-Veloz, A. F., Aldaz-Pachacama, N. R., Mera-Segovia, C. M., Pino-Vaca, D. P., Tapia-Veloz, E. C., \& Vinueza-Veloz, M. F. (2020). Síndrome de Burnout en médicos/as y enfermeros/as ecuatorianos durante la pandemia de COVID-19.

West, C. P., Dyrbye, L. N., Erwin, P. J., \& Shanafelt, T. D. (2016). Interventions to prevent and reduce physician burnout: a systematic review and metaanalysis. The Lancet, 388(10057), 2272-2281.

World Health Organization. (2020). Mental Health and Psychosocial Considerations during the COVID-19 Outbreak. Recuperado em 16 novembro, 2021 , de https://www.who.int/docs/default-source/coronaviruse/mental-health-considerations.pdf. 
Research, Society and Development, v. 10, n. 16, e167101623591, 2021

(CC BY 4.0) | ISSN 2525-3409 | DOI: http://dx.doi.org/10.33448/rsd-v10i16.23591

Wu, Y., Wang, J., Luo, C., Hu, S., Lin, X., Anderson, A. E., ... \& Qian, Y. (2020). A comparison of burnout frequency among oncology physicians and nurses working on the frontline and usual wards during the COVID-19 epidemic in Wuhan, China. Journal of pain and symptom management, 60(1), e60-e65.

Xu, N., Lv, A., Li, T., Li, X., Huang, M., \& Su, Y. (2021). Experiences of healthcare providers during the coronavirus pandemic and its impact on them: protocol for a mixed-methods systematic review. BMJ open, 11(2), e043686.

Yörük, S., \& Güler, D. (2021). The relationship between psychological resilience, burnout, stress, and sociodemographic factors with depression in nurses and midwives during the COVID-19 pandemic: A cross-sectional study in Turkey. Perspectives in psychiatric care, 57(1), 390-398.

Zerbini, G., Ebigbo, A., Reicherts, P., Kunz, M., \& Messman, H. (2020). Psychosocial burden of healthcare professionals in times of COVID-19-a survey conducted at the University Hospital Augsburg. GMS German Medical Science, 18. 